\title{
IMPROVEMENT IN CUTANEOUS SENSIBILITY ASSOCIATED WITH RELIEF OF PAIN
}

\author{
BY \\ P. W. NATHAN \\ From the Neurological Research Unit of the Medical Research Council, the National Hospital, \\ Queen Square, London
}

It is often found that when there is a lesion in the peripheral or the central nervous system causing pain or local tenderness, stimuli applied to the skin of the affected part are not clearly perceived. The clarity of touches with von Frey hairs or cottonwool, the discreteness of pinpricks, the definite perception of when a stimulus is applied and when removedthese may all be absent. Evidence will be presented here showing that when the state of tenderness or pain is removed, such features of the perception of stimulation may be improved, and that this improvement occurs even when a large number of peripheral nerve fibres from the part are put out of action. In fact we may have a condition in which a reduction in the number of conducting nerve fibres results in an improvement in the sensibility of the part. In the most definite examples, there is a diminution in actual sensibility associated with a subjective improvement in perception.

The material illustrating this relation between local pain and cutaneous sensibility is now presented, grouped in the following manner. The first case shows how the local pain and tenderness could be relieved in many ways, and on all occasions where relief occurred there was improvement in subjective sensibility of the part. The second case shows how blocking a peripheral nerve on two occasions may cause a lasting improvement in the quality of the sensibility of the affected part. There follows a group of four cases in which intrathecal injections of phenol in "myodil" or glycerol relieved pain due to cancer; this has been associated with subjective improvement in sensibility. The final group consists of experiments on normal subjects, and it shows how very light stimuli to the skin can be more clearly felt just before nerve conduction fails, this being brought about by occluding the circulation to the limb.

\section{Clinical Material}

In those patients in whom a midline injection of local anaesthetic between the spinous processes relieves a constant pain, it may be found that this $\frac{\bar{s}}{\bar{\sigma}}$ relief is associated with an improvement in the 2 sensibility of the affected dermatome; and this improvement may outlast the duration of the local $\vec{\circ}$ effects of the anaesthetic. A typical case in which:relief of pain has been achieved in this and in various $\vec{\omega}$ other ways is presented first.

Mr. J.H.P.-Some aspects of this case have been reported by Kibler and Nathan (1960). This patienti was complaining of weakness in the right leg and of a tingling and burning pain over the anterior aspect of the right thigh, which was in the distribution of the cutaneogs $N$ branches of the femoral nerve. Eighteen months pe-o viously, Mr. L. S. Walsh had operated for a prolaps intervertebral disc between the second and third lumber vertebrae. At operation it was found that this disc wâs? causing a marked bulge, compressing the dural sace against the lamina of the third lumbar vertebra; several? fragments of the disc were removed. After the operationi $\overrightarrow{0}$ the power of the right leg improved, and the diminuti胃 in sensibility became minimal. The relief of pain lasted only six to eight weeks; and eventually, 18 months after the operation, the patient was referred by Mr. Walsh to the National Hospital. He was then complaining of $a \frac{\partial}{\partial}$ continuous tingling in the skin and a burning pain deep to the skin of the front and the anterolateral part of the right thigh. In the anterior part of the right thigh, there was a raised threshold to all forms of stimulation;을 most pinpricks were there felt merely as pressure, but a very heavy pinprick caused an unpleasant sessation like that of a local electric shock. In the anterolateral part? of the thigh, and in the distribution of the saphenous nerve, there was a slight raising of threshold to pinprick and light touch.

First Experiment.-Two millilitres of $2 \%$ lignocaine were injected just caudal to the spine of the second lumbar vertebra about $4 \mathrm{~cm}$. deep to the skin; it was 3 reckoned that the solution was placed just outside the epidural space.

Within four minutes the patient said that he felt some- $D$ thing hot in the skin supplied by the right lateral femoral cutaneous nerve. Seven minutes after the injection he stated that it felt cold in the whole distribution of the femoral cutaneous nerves; this was the area where heN felt his chronic pain and where there was diminution inN 
sensibility. Three minutes later he reported that the pain was easing and within two minutes it had gone. At this time he found that to rubbing, to touches with von Frey hairs, to pricks with a needle, the sensation was much clearer and more definite than it had been before. $\mathrm{He}$ explained this by saying: "Usually I have to feel these things through a thick sheet of blotting paper. Now the blotting paper has been removed." In his usual state, pinpricks, unless very heavy, were felt only as pressure, though pricks weighted with $140 \mathrm{~g}$. were felt in some places in the anterior and anterolateral aspects of the thigh as painful radiating little electric shocks. After this injection, while the pain was relieved, pinpricks gave a normal sensation of prick, provided they were heavy enough to exceed the raised threshold; the patient said: "I can now feel each one like a proper pin."

Controls for First Experiment.-(a) On the following day $2 \mathrm{ml}$. of normal saline (the same amount as the previous lignocaine) was injected into the same site, the patient being told that the previous day's procedure was being repeated. This caused a few minutes' relief of pain in the back; but it had no lasting effect and no effect on the pain or the sensibility of the right thigh.

(b) Many other injections with $2 \mathrm{ml}$. of lignocaine were carried out. Improvement in the subjective aspects of sensation occurred only when the pain was relieved. Two millilitres of lignocaine relieved the pain only when it was injected between the second and fourth lumbar vertebral spines, into the midline scar, and into the midline $1 \mathrm{~cm}$. cranial to the scar; injections of $2 \mathrm{ml}$. elsewhere had no effect on the pain.

Second Experiment. - A quarter of a millilitre of $6 \%$ saline was injected about $2 \mathrm{~cm}$. deep to the skin in the midline into the middle of the tender scar. At first it caused a very severe pain in the front and the back of the right thigh, and in the back spreading about $1 \mathrm{~cm}$. from the midline bilaterally. As these induced pains passed off, the chronic pain in the front of the thigh was also relieved; he reckoned it went away by about a half. During the period of partial relief of pain, which lasted about 20 minutes, he could perceive in the femoral cutaneous distribution touches with the examiner's finger, with pinprick, with cottonwool, or with hairs, all much more clearly than usually.

It seems to be that any kind of injection that gives relief of pain can improve the sensibility of the tender area.

Third Experiment.-On one occasion an attempt was made to inject the right femoral nerve in the femoral triangle with $1 \%$ lignocaine; but it succeeded only in blocking the muscular branches to the quadriceps and the saphenous nerve. Nevertheless it was found that as soon as the territory of the saphenous nerve was insensitive to touch and to pricking, the pain in the entire limb had gone. It will be realized that the region where the pain and sensory abnormalities were maximal, the area supplied by the anterior femoral cutaneous nerve, was unaffected by this injection, the skin of this part retaining its usual abnormal sensory characteristics. The effects of this injection on relieving the pain lasted from 48 to 60 hours. On this occasion it was also found that the relief of pain was associated with an improvement in the sensibility of the skin in the distribution of the anterior femoral cutaneous nerve: when pinpricks were heavy enough to be felt they no longer had that quality like little electric shocks, but felt discrete like normal pinpricks; they felt more pointed than they otherwise did.

Fourth Experiment.-An electrically driven vibrator was applied to the painful and tender skin of the femoral cutaneous distribution for 15 minutes. It produced local vasodilatation and gave instead of the soreness a pleasant warm feeling. This was associated with a subjective improvement in the appreciation of all stimuli; all pinpricks were felt as such; and rubbing of the skin felt more normal. This improvement lasted about an hour. It could be shown that the improvement was not due to the vasodilatation and rise in local temperature, for it did not accompany these effects when they resulted from local heating of the part with an electric blanket; indeed this local application of heat made both the pain and sensitivity worse.

It is also found that any factors that diminish the local pain and paraesthesiae permanently give a permanent improvement in the appreciation of stimuli.

Mr. G.N.-Certain aspects of this case too have been published by Kibler and Nathan (1960). This patient, who had disseminated sclerosis, had burning pain in the fourth and fifth digits and along the medial border of the left hand. In this area the appreciation of two points, of passive movement, and of vibration was diminished; pinprick felt abnormal: each prick was felt as less sharp than normal, as spreading, burning, and more than normally unpleasant.

The ulnar nerve was injected on two occasions with $2 \%$ lignocaine in the ulnar groove at an interval of 12 days. After the second injection none of the burning pain in the eighth cervical dermatome returned. It was then found that pinpricks in this territory no longer caused the spreading and abnormally unpleasant sensation they had done before; they felt like normal pinpricks, though diminished in intensity compared with pricks applied to the digits of the other hand.

Other cases in which a lasting improvement occurred with permanent relief of pain are those in which the pain of cancer is relieved by the intrathecal injection of phenol in "myodil", as was first described by Maher (1955, 1957). Injections of this solution destroy a large number of nerve fibres of the roots with which the solution makes contact; that this is so has been shown histologically by Marion C. Smith (personal communication). This method of relieving chronic pain usually results in the improvement of sensibility of the affected part, even though it entails a diminution in the number of nerve fibres conducting impulses from the skin.

Mrs. G.C.-This woman attended hospital on account of the pain associated with carcinoma of the left 
Bartholin's gland. An intrathecal injection of $0.75 \mathrm{ml}$. of an $8 \%$ solution of phenol in "myodil" injected between the third and fourth lumbar vertebrae relieved all the patient's pain. Before the injection the patient could not bear to have the upper and outer part of the left buttock touched as it was extremely tender. She had difficulty in walking, she could not lie down on her left side, and she had not sat in a chair for five years as this was too painful. After the injection, the buttock could be touched without causing any pain and it could even be hit hard with the examiner's fist; she could sit comfortably in a chair and could walk without a stick, which she had been unable to do for eight years. On examination, it was found that in this region she could now feel more clearly all stimuli than she had been able to do before. Yet there was a greater degree of loss of the sensation of prick in this area than there had been before the injection, the cold of ice felt less than it had done before, and pressure on this buttock with an algometer showed that the threshold for pain was now raised. Yet when prick with a pin was heavy enough to be felt, it was felt more distinctly than it had been before the injection. Thus in fact there was a diminution in the sensibility of the part; but stimuli adequate to cause a sensation were felt more clearly than previously.

Mrs. G.B.-This woman attended hospital on account of the pain associated with carcinoma corporis uteri. An intrathecal injection of $0.5 \mathrm{ml}$. of a $5 \%$ solution of phenol in glycerol containing $1 / 80$ grain of silver nitrate injected between the second and third lumbar vertebrae relieved all the pain. The patient considered that when she was pricked on this limb she could feel the pricks better than before the injection. Yet it was found that in fact there was a level between the first and second lumbar dermatomes where pinprick became sharper and more painful and where ice was felt as colder. Thus the evidence showed that below the first lumbar dermatome there was in fact a diminution in the number of conducting nerve fibres; and yet the patient felt the stimulation more clearly in this area in which there was a slight diminution in sensibility.

Miss M.B.-This woman attended hospital on account of the pain associated with carcinoma cervicis uteri. An intrathecal injection of $0.5 \mathrm{ml}$. of a $7.5 \%$ solution of phenol in "myodil" injected between the twelfth thoracic and first lumbar vertebrae relieved all the pain. Although objectively no change was found in the area or in the quality of her sensibility after this injection, the patient insisted that what she could feel she could feel more clearly than before; she affirmed that the sensation on having the right lower limb rubbed was more nearly normal after this injection than it had been before.

Mrs. D.R.-This woman attended hospital on account of the pain associated with carcinoma cervicis uteri. An intrathecal injection of $1.5 \mathrm{ml}$. of a $7.5 \%$ solution of phenol in "myodil" between the fourth and fifth lumbar vertebrae was given. This reduced her pain a great deal, she estimating that $90 \%$ of it had been relieved. Before the injection the sensibility to pinprick was diminished in the left fifth lumbar dermatome; following it, she could feel pinprick more clearly here than before; the pricks felt sharper and each prick felt less diffuse than it had done before. Subjectively the foot and toes felt less numb than before the injection, and the limb "felt as though it had more life in it." Objectively, no changes in sensibility could be found.

Thus in these four patients, in whom the intrathecal injection of a solution of phenol destroyed a large number of nerve fibres and relieved the pain, there was an improvement in their perception of stimuli; although there was no lowering of threshold to stimulation, indeed the contrary was sometimes the case, the patients felt that which they could feel more clearly, and the stimulations were felt more discretely.

A similar improvement in the ability to feel stimulations associated with a reduction in the number of functioning peripheral nerve fibres can $\overrightarrow{0}$ be induced experimentally in normal subjects. When the blood supply to a nerve or limb is occluded, paraesthesiae occur, and during the time of these paraesthesiae, there is interference with the perception of exteroceptive stimuli. This inability to feel i slight stimuli can be demonstrated by applying a light von Frey hair. The phenomenon being described here is more difficult to bring out; it found only when an even lighter hair, one of subs 음 threshold intensity, is used as the stimulus. The@ there may be found an increased ability to feel sua $\bar{T}$ very light stimuli at a time when all conduction i\& the $\mathbf{A}$ group of fibres is about to fail. It is know that at this time the conducted action potential the sensory fibres with the lowest threshold reduced to about a half of its original size. The ability to feel the stimuli better at this time is not striking when the number of stimulations felt is recorded; but if attention is directed to the quality of the sensation, then nearly all subjects report that just after the time to numbness comes on they feel this very slight stimulus as heavier, as broader in area, as longer lasting, as generally easier to feel, than they had done before.

The quantitative aspect is illustrated in Fig. 1. This graph is the mean of 10 experiments performed on 10 normal male subjects, the stimulus being a nylon No. 1 hair. This hair was felt less than $20 \%$ 음 of applications under normal conditions. From Fig. 1 it can be seen that just before the stimulus ceases to be felt altogether, for a short period of time it is felt more often than at any period before, in- 을 cluding the control period before the occlusion of the circulation; just as the time to numbness arrived it was felt $34 \%$ of its times of application, whereas in the control period it was felt $17 \%$ of times. The $\mathrm{N}$ period of improved perception lasted three minutes. N In two of these subjects on another occasion the hair 


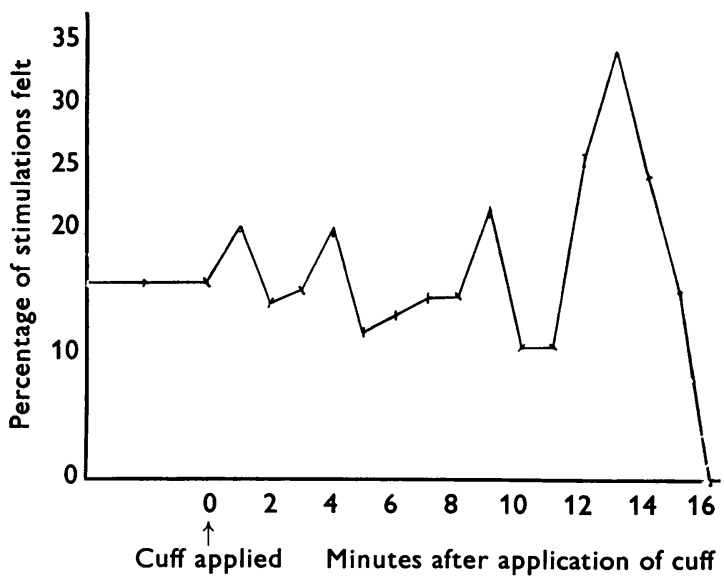

FIG. 1.-Average of 10 normal subjects; cuff on arm; stimulus nylon No. 1 hair.

was applied 10 times for every minute of a period of 30 minutes, without occlusion of the circulation, so as to rule out any changes in perception due to factors such as tiring, boredom, learning, or practice.

This experimentally induced improvement in perception of stimulation with a reduction in the number of conducting nerve fibres is not restricted to tactile stimulation; it is also found for painful stimulation.

Protocol of Experiment.-A block was applied to the ulnar nerve by a subject leaning his weight on his flexed upper limb, in such a position that a $4 \mathrm{~cm}$. length of the ulnar nerve was compressed. The temperature of the skin of the fifth digit was kept at $33-34^{\circ} \mathrm{C}$. throughout. The stimulus consisted of a shock applied by means of the sparker (Morton's modification of the instrument described by Bishop, described in a paper by Nathan, 1958) to the dorsum of the fifth digit immediately proximal to the nail.

This experiment is illustrated in Fig. 2. It can be seen that at the thirteenth minute after application of pressure to the ulnar nerve, only three of 10 stimulations felt painful; over the next two minutes there was a recovery in the ability to feel the painful stimulation; at the sixteenth minute, no more pain was felt.

This apparent recovery of sensibility occurring at a time when many of the nerve fibres must have ceased to conduct may be due to an increased prominence of the signal; possibly the signal to noise ratio in the central nervous system is increased. This phenomenon would seem to be the opposite of the inability to feel exteroceptive stimulation during periods of paraesthesiae, as has been described by Gilliatt and Grahame Wilson (1954) and Nathan (1958). In that phenomenon the signal is completely submerged among the masses of impulses pouring into the central nervous stystem; in the present phenomenon, the signal stands out clearly amid the silence from the absence of other impulses arriving at the central nervous system.

\section{Discussion}

The most striking observation reported here is that the input from the periphery may be diminished and yet the perception of stimulation can be improved. This improvement occurs with either a raising or with no change of the sensory threshold. Once the pain or paraesthesiae have been taken away, the subject can make better use of the fewer remaining nerve fibres. Or, in other words, in the presence of local pain, tenderness, or paraesthesiae, the accurate perception of stimuli is rendered difficult. This phenomenon is not always found. In some patients the vibrator applied to the tender skin has relieved the pain, and yet there has been no improvement in sensibility; if there has been any objective change, the threshold has been slightly raised, and stimuli that were felt before the vibration might not be felt after it.

A phenomenon probably related to that reported here has been demonstrated and investigated by Gilliatt and Grahame Wilson (1954) and by Nathan (1958). They showed that when large cutaneous fibres are firing impulses into the central nervous

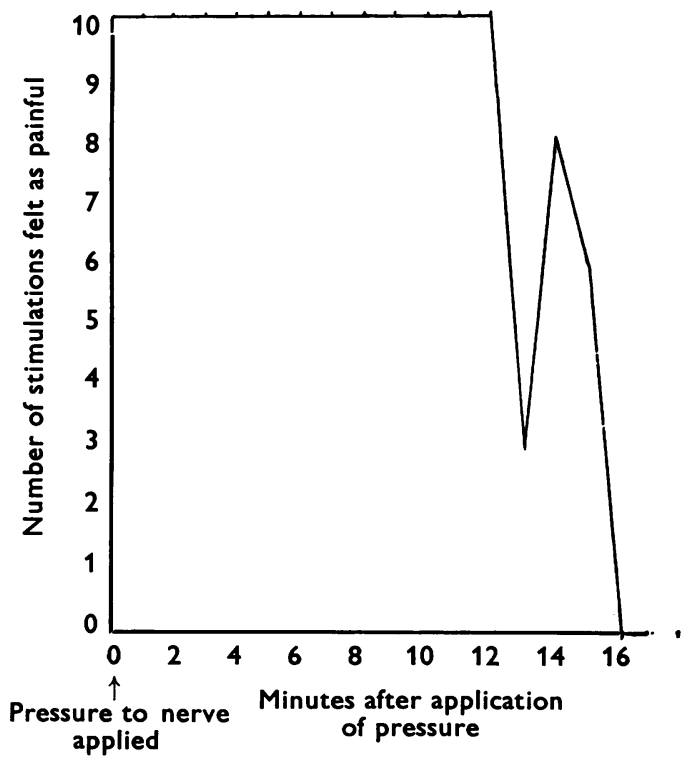

Fig. 2.-A normal subject; cuff on arm; stimulus sparker at $24 \mathbf{v}$. 
system, the perception of stimuli in the territory of the active nerve fibres is impaired. Nathan further showed that this can occur even when the spontaneously arising impulses reaching the central nervous system are giving rise to no sensation. It may be that the impulses subserving the sensation of pain felt in the local region impair the perception of peripheral stimulation in a similar way. In both cases there would seem to be a convergence of the two lots of impulses. Where in the central nervous system this convergence might occur is unknown; one could conceive of it occurring at the level of the spinal cord, of the reticular substance, or any place where afferent or sensory functions are integrated.

\section{Summary}

In patients in whom a lesion is causing localized pain, stimuli applied to the skin of the region where the pain is felt may be poorly perceived. Any measure that relieves the pain and local tenderness, including measures that block some of the afferent fibres from the area, may improve the perception of stimuli. A similar phenomenon is described in normal subjects, when the occlusion of the circulation to a nerve is induced; just before sensibility for touches and pricks fails, for two or three minutes ${ }^{\infty}$ these stimuli are felt more clearly than before. It ${ }^{D}$ is suggested that in both cases the signal to noise ratio is increased when many of the afferent nerve fibres are prevented from conducting impulses into the central nervous system.

I take this opportunity of thanking the physicians and surgeons of the National Hospital, Queen Square, who were kind enough to put their patients at my disposal for this investigation, and Dr. E. A. Carmichael for his encouragement and for the facilities to do this work.

\section{REFERENCES}

Gilliatt, R. W., and Grahame Wilson, T. (1954). J. Neurol. Neurosurg. Psychiat., 17, 104.

Kibler, R. F., and Nathan, P. W. (1960). Ibid., 23, 91

Maher, R. M. (1955). Lancet, 1, 18 .

Nathan, P. W. (1958). J. Neurol. Neurosurg. Psychiat., 21, 12. 